\title{
Mujer y deporte en México. Hacia una igualdad sustancial Women and sport in Mexico. Towards a substantial equality
}

Zitlally Flores Fernandez

Universidad Juárez del Estado de Durango (México)

\begin{abstract}
Resumen. Este artículo presenta un análisis de la situación de la mujer en el deporte en México con el objetivo de determinar si existen desequilibrios con respecto a los hombres, así como el análisis de la política pública nacional en materia de cultura física y deporte respecto a las acciones implementadas para disminuir las brechas de desigualdad. La orientación metodológica es de orden teórico, los métodos empleados son el lógico deductivo, exegético y analítico. Los principales resultados de las encuestas realizadas por el INEGI sobre la práctica deportiva y ejercicio físico de las mujeres en los años 2013 al 2016 y la participación de la mujer en el deporte de competición dan muestra que la política pública implementada ha sido ineficiente e ineficaz para disminuir las brechas de desigualdad con respecto a los hombres, se concluye que es necesaria la priorización de acciones positivas concretas para acelerar la participación de la mujer en el deporte en condiciones de igualdad.
\end{abstract}

Palabras claves: Mujer, deporte, derecho, igualdad sustancial, políticas públicas.

\begin{abstract}
This paper presents an analysis of the situation of women in sports in Mexico with the objective of determining if there are imbalances with respect to men, as well as an analysis of the national public policies on physical culture and sport regarding actions implemented to reduce the gaps in inequality. The methodological orientation lays on a theoretical order, the used methods are logical deductive, analytical, and exegetical. The main results of the surveys carried out by the INEGI on women's sports practice and physical exercise between the years 2013 and 2016, and on women's participation in sports competitions, show that the public policies implemented have been inefficient and ineffective in reducing the gaps in inequality with respect to men. In conclusion, it is necessary to prioritize concrete positive actions to expedite women's participation in sport on an equal level.
\end{abstract}

Key words: Woman, sports, law, substantive equality, public policy.

\section{Introducción}

El derecho a la práctica del deporte está reconocido constitucionalmente en México, el artículo $4^{\circ}$ establece que: «Toda persona tiene el derecho a la cultura física y a la práctica del deporte correspondiéndole al Estado su promoción, fomento y estímulo conforme a las leyes en la materia.» El Estado debe asegurarle a la mujer las mismas oportunidades de participar activamente en el deporte y la educación física, así lo contempla la Convención sobre la eliminación de todas formas de discriminación contra la mujer (CEDAW) en sus artículos 10 y 13. Sin embargo, cuando en los hechos existen marcadas desigualdades no es suficiente con garantizar un trato idéntico al de los hombres ya que, los roles y estereotipos de género atribuidos a mujeres y hombres influyen significativamente en las posibilidades y oportunidades efectivas para la práctica del deporte, la educación física aún sigue estando ligada a la masculinidad, en dónde algunas chicas se perciben menos valoradas y con menos oportunidades de participación (Beltrán, Sierra, Jiménez, GonzálezCutre, Martínez \& Cervelló, 2017). Esto ha motivado a realizar propuestas encaminadas a resolver los estereotipos de género en las clases de educación física a través de la adaptación de las normas y reglas del fútbol que permitan brindan una igualdad de trato y oportunidades entre el alumnado (Rodríguez \& Miraflores, 2018), asimismo, los estereotipos de género y el rol femenino actual parecen tener un peso específico en los motivos de abandono de las deportistas (Isorna, Felpeto, Alonso, Gómez \& Rial, 2019).

Desde una perspectiva jurídica es necesario distinguir entre la igualdad ante la ley y la igualdad sustancial. La igual-

Fecha recepción: 23-03-19. Fecha de aceptación: 19-08-19 Zitlally Flores Fernandez

zitlallyfloresfernandez@gmail.com dad ante la ley se proyecta en diversas facetas: a) igualdad en la norma jurídica general, obligando al legislador a no cometer distinciones arbitrarias o irrazonables; b) igualdad frente a la norma jurídica, vinculando al órgano encargado de aplicarla; c) la igualdad de derechos, significa que todos los hombres son titulares por igual de ciertos derechos, calificados como derechos humanos (Didier, 2016).

Por su parte, la igualdad sustancial, real o de hecho tiene por objetivo la realización práctica de ésta a través de acciones específicas para quienes no están en la misma situación de ventaja, lo que puede entrañar un tipo de discriminación temporal para compensar hábitos y prácticas desventajosas (Postigo, 2005). Se refiere, por lo tanto, a la obligación del Estado de implementar acciones positivas en beneficio de grupos vulnerables como lo es el de las mujeres, para remover los obstáculos que impiden el ejercicio real y efectivo de determinado derecho, en este caso al deporte. Por lo que una de las principales obligaciones del Estado es la de identificar los grupos que en un determinado momento histórico necesitan atención prioritaria o especial e incorporar en sus planes de acción medidas concretas para proteger a esos grupos (Amabrovich, 2016).

\section{Objetivos}

Analizar la situación de la mujer en México en la práctica de actividades físicas y deportivas, con base en las encuestas realizadas por el Instituto Nacional de Estadística y Geografía (INEGI) en los años 2013 al 2016, así como la participación de la mujer mexicana en el deporte de alto rendimiento, que permita vislumbrar desigualdades con respecto a los hombres.

Analizar si el Estado mexicano ha incorporado en su política pública en materia de cultura física y deporte acciones concretas para proteger el derecho de las mujeres a la práctica del deporte. 
El estudio pretende dar cuenta que no existe una política pública nacional con proyectos específicos encaminada a rectificar los desequilibrios existentes en materia de deporte.

\section{Método}

El presente estudio es de carácter cualitativo, la metodología seguida se encuadra en el paradigma interpretativo (hermenéutico), la orientación es de orden teórico, los métodos empleados son el exegético a partir del contenido del marco legal del derecho a la cultura física y el deporte que sustenta al trabajo de investigación y el analítico para procesar la información obtenida de las encuestas realizadas por el INEGI en el periodo comprendido del 2013 al 2016, así como los datos obtenidos sobre la participación de la mujer en el deporte de alto rendimiento en México.

Partiendo de la premisa de la obligación del estado mexicano de implementar acciones concretas en beneficio de las mujeres que les permita el ejercicio real del derecho a la práctica del deporte, se analiza la política pública nacional implementada del 2013 al 2018 y mediante el método lógico deductivo se determina si el estado cumple con su obligación constitucional y si la política pública implementada ha contribuido a disminuir las brechas de desigualdad con respecto a los hombres.

\section{Resultados}

\section{Situación de la mujer en la práctica de actividades físico-deportivas}

Las investigaciones respecto al nivel de actividad física en México, iniciaron hasta 1996 cuando el Instituto Nacional de Estadística y Geografía (INEGI), empieza a captar información relacionada con el nivel de actividad física en el país de hombres y mujeres de quince y más años de edad. Posteriormente, en noviembre de 2013, se capta por segunda ocasión información referente al tema, con datos sobre la participación de hombres y mujeres de dieciocho y más años de edad en la práctica de algún deporte o la realización de ejercicio físico en su tiempo libre. (MOPRADEF, 2017).

A continuación, se presenta una comparación de los principales resultados de las encuestas realizadas por el INEGI mediante el Módulo de Práctica Deportiva y Ejercicio Físico de los años 2013 al 2016, respecto a la población de 18 y más años de edad del agregado urbano de 32 áreas de 100 mil y más habitantes, sobre la práctica de algún deporte o ejercicio físico en su tiempo libre, esto desde una perspectiva diferenciadora de género y de edad, dado que, el proceso de socialización de cada persona influye en sus actitudes y comportamientos ante el deporte y estas variables son fundamentales para comprender las relaciones sociales, las estructuras y formas que se derivan de las mismas (García, Puig \& Lagardera, 2009), conocer los motivos que llevan a las mujeres a realizar actividades físicas o deportivas es de gran importancia para el diseño de programas de intervención, en su estudio, Pérez, Castaño, Sánchez, Rodríguez, De Mena, Fuentes, \& Castaño, (2018) refieren que las motivaciones que llevan a la práctica del fútbol a las mujeres no se mantienen estables a lo largo de los distintos años, por lo que las políticas públicas en materia deportiva deberán partir de una perspectiva diferenciadora no sólo del género sino también de la edad.

En México, en el 2013 el 54.4\% de los hombres eran activos físicamente, esto quiere decir que acumularon un mínimo de 150 minutos semanales de actividad física aeróbica moderada, o bien 75 minutos de actividad física aeróbica vigorosa cada semana, o bien una combinación equivalente de actividades moderadas y vigorosas, este porcentaje de hombres activos se mantuvo durante 2014 y 2015 sin una variación significativa (54.8\%, 54.2\%, respectivamente), para el 2016, el número de hombres activos bajó a 47.7\%. Por su parte, el porcentaje de mujeres activas ha sido menor con respecto a los hombres, en el 2013, el 45.6\% de la población femenina era activa físicamente y al igual que con los hombres esta tendencia se mantuvo sin una variación significativa en los años 2014 y 2015 (45.2\%, 45.8\%, respectivamente), para el 2016, el número de mujeres activas bajó a 36.7\% significando una diferencia de más de 10 puntos porcentuales con relación a los hombres. El siguiente gráfico muestra la proporción de hombres y mujeres activos en el periodo de 2013 a 2016.

Como se puede apreciar, la prevalencia de inactividad física es significativamente mayor en las mujeres. En nuestra sociedad se han asignado sistemas de valores, pautas de comportamiento y roles diferentes para mujeres y hombres, estos valores y normas adquiridos durante el proceso de socialización han influido, al deporte se le ha asociado con valores considerados masculinos (García, et. al, 2009). Alos hombres se les relaciona con estereotipos de fortaleza, insensibilidad, proveedor, jefe de familia, orientados hacia la competitividad, mientras que, a las mujeres con estereotipos de belleza, seducción, dulzura, abnegación, sacrificio que exaltan el matrimonio y la maternidad (Guichard, 2015).

En la siguiente tabla se muestra la proporción de hombres y mujeres activos por grupo de edad.

Se observa que en el rango de 18 a 24 años tanto hombres como mujeres son más activos físicamente con excepción del 2016, en donde llama la atención que el grupo de mujeres más jóvenes no representa la mayor proporción de mujeres activas, siendo las del rango de treinta y cinco a 44 años quienes presentan esta condición.

La tabla muestra que cada año, es menor el número de mujeres jóvenes de entre dieciocho a 24 años activas físicamente en México, situación que resulta preocupante más

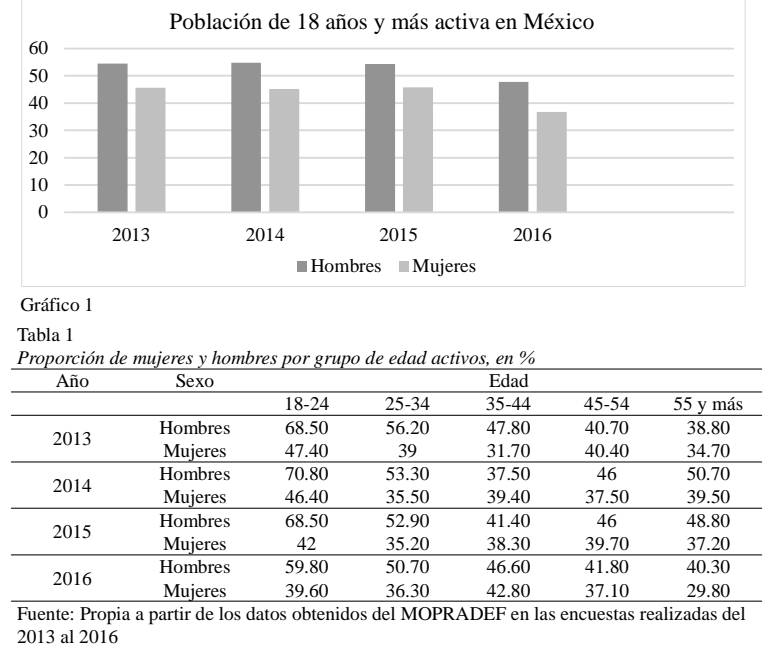


aún si consideramos que de acuerdo a la Encuesta Nacional de Salud (ENSANUT, 2016) la prevalencia de sobrepeso en adolescentes de sexo femenino ha incrementado en los últimos años y la prevalencia combinada de sobrepeso y obesidad en 2016 en adultos de 20 años y más es de 72.5\% siendo más alta la prevalencia tanto de sobrepeso como de obesidad y de obesidad mórbida en el sexo femenino. Resulta por lo tanto necesario implementar acciones tendientes a lograr una mayor participación de la mujer joven en actividades físicas, medidas que deben ser implementadas desde el ámbito escolar, Beltrán et. al (2017), al analizar las diferencias según género en el tiempo empleado por adolescentes en actividad sedentaria y actividad física en diferentes horarios del día, encontraron que las chicas presentan una mayor actividad sedentaria, estos autores plantean una serie de recomendaciones para la promoción de la actividad física en las chicas, como obtener información, a principio de curso, sobre las preferencias de actividad física y deporte de chicas y chicos de modo que el currículum pudiera estar equiparado en función de las preferencias de los alumnos de ambos géneros.

En lo referente al grupo de edad de 55 y más hay una disminución considerable del número de mujeres activas físicamente, representando para el 2016 sólo el 29.80\%. Estudios en España sobre diferencias de género en las motivaciones para practicar actividades físico deportivas en la vejez, (Martín, Moscoso \& Pedrajas, 2013), han aportado datos respecto al fuerte componente extrínseco relativos a la mejora y el mantenimiento de la forma física relacionada con la salud y otros de carácter intrínseco, como el gusto por la actividad, mismo que debieran ser considerados como elementos de referencia para el diseño de la política pública en materia de cultura física y deporte para personas mayores.

La siguiente tabla muestra las desigualdades entre hombres y mujeres de 18 a 24 años de edad en el periodo comprendido de 2013 a 2016 en donde se puede apreciar que no se ha podido superar la brecha de más de 20 puntos porcentuales de hombres activos con relación a las mujeres.

\begin{tabular}{|c|c|c|c|c|c|}
\hline \multicolumn{6}{|c|}{ Mujeres } \\
\hline & Año & Activos & Inactivos & $\begin{array}{c}\text { Que alguna vez realizaron } \\
\text { actividad física }\end{array}$ & $\begin{array}{l}\text { Que nunca realizaron } \\
\text { actividad física }\end{array}$ \\
\hline & 2013 & 47.4 & 52.6 & 74.2 & 25.8 \\
\hline & 2014 & 46.4 & 53.6 & 73.51 & 26.5 \\
\hline & 2015 & 42 & 58 & 85.9 & 14.1 \\
\hline & 2016 & 39.6 & 60.4 & 77.4 & 22.6 \\
\hline \multicolumn{6}{|l|}{ Hombres } \\
\hline & 2013 & 68.5 & 31.5 & 77.5 & 22.5 \\
\hline & 2014 & 70.8 & 29.2 & 80 & 20 \\
\hline & 2015 & 68.5 & 31.5 & 81.1 & 18.9 \\
\hline & 2016 & 59.8 & 40.2 & 97.6 & 2.4 \\
\hline
\end{tabular}

El nivel de escolaridad juega un papel importante, a mayor nivel educativo se incrementa el porcentaje de la población que declaró practicar una actividad físico-deportiva. Para el 2016, el 49\% de las mujeres activas contaba con al menos un grado de educación superior, mientras que de las mujeres inactivas que nunca realizaron actividad física, el 57.4\% no contaban con educación básica terminada. Los años de escolaridad se asocian al nivel de actividad física en tiempo libre, las personas con menos estudios realizan menos actividad física de tiempo libre (Tarducci, Barengo, Morea, Gárgano, Gandini, Paganini \& Bardach, 2016). Por otra parte, de las mujeres económicamente activas el 64\% son inactivas físicamente, la principal razón que mencionaron de no practicar actividades físico-deportivas o motivo de abandono fueron la falta de tiempo, el cansancio por el trabajo y problemas de salud.

Con relación a la participación de la mujer en el deporte de alto rendimiento, un estudio realizado por el Instituto Nacional de las Mujeres INMUJERES y la Comisión Nacional de Cultura Física y Deporte CONADE (2005), refiere que la situación de la mujer en el deporte mexicano no es la ideal, pero tampoco es particular, pues forma parte de una desigualdad social donde las mujeres se han encontrado en desventaja respecto a los hombres, en donde siguen vigentes los estereotipos sociales sobre la mujer en el renglón de las capacidades físicas, su rol social y la forma en que se perciben ellas mismas ante los retos de la práctica deportiva. Como lo refiere Marta Lamas (1996) debemos reconocer que una sociedad desigual tiende a repetir la desigualdad en todas sus instituciones y en este caso el ámbito deportivo no ha sido la excepción, aunado a que, la práctica deportiva competitiva exige un nivel de compromiso más elevado para el deportista, por lo que la incidencia de abandono es aún más preocupante en el caso de las mujeres (Isorna, et. al, 2019). La desventaja de las mujeres mexicanas en el entorno deportivo olímpico es significativamente importante, la siguiente tabla da muestra de ello.

\begin{tabular}{|c|c|c|c|c|}
\hline Olimpiadas lugar y año & Mujeres & Hombres & Total & $\%$ mujeres \\
\hline Los Ângeles 1932 & 2 & 71 & 73 & 2.7 \\
\hline Berlín 1936 & 0 & 35 & 35 & 0 \\
\hline Londres 1948 & 7 & 82 & 89 & 7.9 \\
\hline Helsinki 1952 & 2 & 64 & 66 & 3 \\
\hline Melibour 1956 & 3 & 22 & 25 & 12 \\
\hline Roma 1960 & 7 & 61 & 68 & 10.3 \\
\hline Tokio 1964 & 4 & 85 & 89 & 4.5 \\
\hline México 1968 & 46 & 266 & 312 & 14.7 \\
\hline Munich 1972 & 23 & 154 & 177 & 13 \\
\hline Montreal 1976 & 6 & 97 & 103 & 5.8 \\
\hline Moscú 1980 & 9 & 36 & 45 & 20 \\
\hline Los Ángeles 1984 & 21 & 78 & 99 & 21.2 \\
\hline Seúl 1988 & 20 & 71 & 91 & 22 \\
\hline Barcelona 1992 & 31 & 106 & 137 & 22.6 \\
\hline Atlanta 1996 & 28 & 81 & 109 & 25.7 \\
\hline Sidney 2000 & 27 & 51 & 78 & 34.6 \\
\hline Atenas 2004 & 52 & 62 & 114 & 45.6 \\
\hline Beijin 2008 & 42 & 43 & 85 & 49.4 \\
\hline Londres 2012 & 37 & 61 & 98 & 37.8 \\
\hline Río 2016 & 47 & 85 & 132 & 35.6 \\
\hline Total & 414 & 1611 & 2025 & 19.42 \\
\hline
\end{tabular}

La primera participación de la mujer mexicana en Juegos Olímpicos fue en Los Ángeles 1932, a partir de este año y hasta los juegos de Río 2016 la mujer ha representado tan sólo el $19.42 \%$. Si bien es cierto que la participación de la mujer ha ido en aumento (alcanzándose en Beijin 2008 el mayor porcentaje de representación femenina, 49.4\%) el deporte de alto nivel femenino representa la lucha constante por obtener la igualdad frente al masculino, en dónde el sistema patriarcal imperante opone serios obstáculos para la incorporación de la mujer, agudizándose en los deportes considerados masculinos en dónde las dificultades son mayores (Puig \& Soler, 2004), en México por ejemplo, la participación femenina en Juegos Olímpicos se ha concentrado en natación, atletismo, clavados, nado sincronizado y gimnasia, el nado sincronizado y la gimnasia son disciplinas estereotipadas con la «feminidad» (Transversalización de la Perspectiva de Género en el Ámbito Deportivo Mexicano, 
2016). La influencia de los estereotipos de género en la sociedad y la enseñanza, terminan por generar diferencias tanto de intereses como de motivaciones, así como en el grado de práctica de ciertas modalidades deportivas. (Isorna, et. al., 2019).

Ante este panorama de desigualdad sustancial, el Estado tiene la obligación de adoptar medidas especiales que incluyan políticas activas tendientes a disminuir las barreras tanto internas como externas que impiden la participación de la mujer en las actividades físicas y en el deporte de competencia, obstáculos que enfrentan las mujeres como la tradición cultural eminentemente masculina de muchas modalidades deportivas, los conflictos de rol o de género, las diferencias en retribución económica según el género, la falta de modelos femeninos en los medios de comunicación o la escasa representación de las mujeres en los órganos de decisión (Isorna, et. al., 2019), son algunos de los obstáculos que debieran de ser atendidos mediante políticas públicas si se busca que las mujeres tengan oportunidades reales y efectivas para la práctica de actividades físicas y deportivas.

\section{Política pública en México en materia deportiva}

El Plan Nacional de Desarrollo (PND) es el instrumento en el que se precisan los objetivos nacionales, estrategias y prioridades del desarrollo integral y sustentable del país, el PND 2013-2018 establece cinco metas nacionales: México en Paz, México Incluyente, México con Educación de Calidad, México Prospero y México con Responsabilidad Global, cuenta con tres estrategias transversales; democratizar la productividad, gobierno cercano y moderno, perspectiva de género.

El deporte se incluye en la meta de educación de Calidad por considerar que la oferta deportiva es un medio valioso e imprescindible para consolidar una educación integral. Se establece el objetivo de promover el deporte de manera incluyente para fomentar una cultura de salud, esto mediante dos estrategias: Crear un programa de infraestructura deportiva y Diseñar programas de actividad física y deporte diferenciados para atender las diversas necesidades de la población. Esta estrategia contempla 5 líneas de acción:

1. Crear un programa de actividad física y deporte tendiente a disminuir los índices de sobrepeso y obesidad.

2. Facilitar la práctica deportiva sin fines selectivos con un enfoque que promueva la adquisición de valores para el trabajo en equipo, respeto a las reglas y obtención del éxito mediante el esfuerzo individual y colectivo.

3. Estructurar con claridad dos grandes vertientes para la práctica de actividades deportivas: deporte social y deporte de representación.

4. Facilitar el acceso a la población con talentos específicos al deporte estrictamente selectivo.

5. Llevar a cabo competencias deportivas y favorecer la participación de la población en competencias municipales, estatales, nacionales e internacionales.

Estas líneas de acción no incluyen de manera explícita acciones orientadas hacia las mujeres, a garantizarles el derecho a la práctica del deporte y evitar que las diferencias de género sean causas de desigualdad, exclusión o discriminación.

La estrategia transversal de Perspectiva de género tiene como objetivo que se incorpore plenamente la perspectiva de igualdad de género en las políticas públicas, programas, proyectos e instrumentos compensatorios como acciones afirmativas de la Administración Pública Federal (DOF 30 agosto 2013), para ello se creó el Programa Nacional para la Igualdad de Oportunidades y no Discriminación contra las Mujeres 2013-2018 (PROIGUALDAD), este programa incluye en la estrategia 4.5 Fortalecer las actividades artísticas, culturales y deportivas con acciones afirmativas hacia las mujeres y niñas y contempla las siguientes líneas de acción:

4.5.1 Procurar la construcción y adecuación de espacios escolares deportivos con infraestructura y servicios que contemplen las necesidades de las mujeres.

4.5.2 Fortalecer la formación deportiva de niñas, adolescentes y jóvenes que incremente su participación en competencias nacionales e internacionales.

4.5.3 Diversificar las actividades deportivas de las mujeres en las escuelas de educación básica y media superior.

4.5.4 Promover que todos los programas de fomento deportivo se diseñen con perspectiva de género.

4.5.5 Fomentar las actividades deportivas para mujeres adultas en los municipios.

Asimismo, la línea de acción 4.7.7 contempla promover actividades de deporte y bienestar físico de acuerdo a requerimientos específicos de las adultas mayores.

Por su parte, el Programa Nacional de Cultura Física y Deporte 2014-2018 (DOF 30 abril 2014) establece en su estrategia 5.3 Impulsar el desarrollo social a través del deporte, en especial el desarrollo femenino y de las personas con discapacidad mediante dos líneas de acción:

1. Poner en marcha un proyecto de comunicación que promueva la participación de las mujeres en el deporte.

2. Promover proyectos específicos para la mujer y las personas con discapacidad.

Si bien el tema del deporte está presente en PROIGUALDAD, lo cierto es que se muestra ambigüedad en las líneas de acción, tanto en el Programa Nacional de Cultura Física y Deporte como en PROIGUALDAD no se incluyen acciones referentes al deporte de alta competición en mujeres.

\section{Discusión}

Al analizar los instrumentos que conforman la política pública en materia de cultura física y deporte se ha encontrado que:

Las líneas de acción del PND 2013-2018 no incluyen de manera explícita acciones orientadas hacia las mujeres, a garantizarles el derecho a la práctica del deporte y evitar que las diferencias de género sean causas de desigualdad, exclusión o discriminación.

Las líneas de acción del Programa Nacional de Cultura Física y Deporte no contemplan proyectos concretos para atender a grupos específicos de mujeres como son jóvenes, adultas mayores, mujeres económicamente activas, mujeres de alta competición, etc.

El Programa Nacional de Cultura Física y Deporte no define indicadores que den cuenta de la incorporación de la perspectiva de género a corto, mediano o largo plazo.

Los instrumentos antes descritos carecen de estrategias 
que atiendan la problemática identificada en las encuestas aplicadas por el INEGI a través del MOPRADEF y a la situación de la mujer en el deporte de alta competición.

Los principales resultados de las encuestas realizadas por el INEGI sobre la práctica deportiva y ejercicio físico de las mujeres en los años 2013 al 2016 dan muestra que la política pública implementada ha sido ineficiente e ineficaz para disminuir las brechas de desigualdad con respecto a los hombres, las mujeres en México hoy en día son menos activas físicamente que en años anteriores, por lo que para lograr la igualdad sustantiva entre hombres y mujeres en materia de cultura física y deporte, es necesario la priorización de acciones positivas para acelerar la participación de la mujer en condiciones de igualdad.

\section{Conclusiones}

La declaración de Brighton sobre la mujer y el deporte establece en sus principios que: «los recursos, el poder y la responsabilidad han de ser repartidos justamente y sin discriminación sexual, pero cada asignación ha de rectificar los desequilibrios injustificables entre las ventajas disponibles para las mujeres y para los hombres.» Las políticas públicas en materia de cultura física y deporte deben tomar en cuenta las barreras tanto internas como externas que impiden la participación de la mujer en las actividades físicas y en el deporte de competencia, considerar las desigualdades fácticas manifiestas en los resultados de las encuestas aquí presentadas para rectificar los desequilibrios injustificables entre las ventajas disponibles para las mujeres y para los hombres, por lo tanto, el estado mexicano debe rendir cuentas sobre las acciones que está implementando y porqué considera que dichas acciones son las más apropiadas.

La política pública «exige un proceso racional, informado y comprometido de selección y definición de problemas prioritarios, a partir de los medios efectivamente disponibles para solucionarlos» (Merino, 2005, p. 9), de acuerdo con Aguilar (2010, p. 29) lo distintivo de la política pública es el hecho de «integrar un conjunto de acciones estructuradas, estables, sistemáticas, que representan el modo en el que el gobierno realiza de manera permanente y estable las funciones públicas y atiende los problemas públicos.» Se coincide con Valdez Méndez (2015, p. 50), cuando afirma que: «en México no existe al menos en el diseño, una política pública de fomento a la actividad física, sino acciones generales, apegadas al diseño institucional particular y con falta de distribución específica de competencias...» Abordar las situaciones que inciden en el bienestar de las personas desde una perspectiva de género implica ver la realidad desde un enfoque diferente que permite percibir y comprender la manera en que las normas sociales crean desigualdades entre hombres y mujeres que se traducen en desventajas (Vargas \& Gambara, 2012). Los estilos de vida es el resultado de un conjunto de factores tanto individuales como sociales, las medidas parciales tendrán logros limitados, por lo que será necesario el diseño de planes globales en los que se involucren diversos agentes sociales (Nuviala, Grao, Fernández, Alda, Burges \& Jaume, 2009). La incorporación de la perspectiva de género en las políticas públicas en materia de cultura física y deporte implica un proceso que incluya acciones concretas y reales bien planificadas tendientes a lograr una mayor participación de la mujer de distintas edades en la práctica de actividades físicas en el tiempo libre, así como en su participación en el deporte de alta competición, sólo así se podrá logar una igualdad sustancial.

\section{Referencias}

Aguilar, L. (2012). Política pública. México: Siglo XXI.

Amabrovich, V. (2006). Una aproximación al enfoque de derechos en las estrategias y políticas de desarrollo. Revista de la CEPAL, 88, 35-50.

Beltrán, V., Sierra, A., Jiménez A., González-Cutre, D., Martínez, C., \& Cervelló, E. (2017). Diferencias según género en el tiempo empleado por adolescentes en actividad sedentaria y actividad física en diferentes segmentos horarios del día. RETOS. Nuevas tendencias en Educación Física, Deporte y Recreación,31 (1), 3-7.

CEAMEG. (2016). Proyecto de documento transversalización de la Perspectiva de Género en el Ámbito Deportivo Mexicano. Recuperado de https:// app.vlex.com/\#vid/proyecto-documento-transversalizacion-perspectiva679108957

Declaración de Brighton sobre la Mujer y el Deporte. Recuperado de http:// www.csd.gob.es/estaticos/myd/declaracion-brighton.pdf

Didier, M. (2012). La exigibilidad judicial de los derechos sociales básicos: un imperativo del principio de igualdad. Persona y derecho, (66), 81-97.

Encuesta Nacional de Salud (2016) Recuperado de http:// www.promocion.salud.gob.mx/dgps/descargas1/doctos_2016/ ensanut_mc_2016-310oct.pdf

García, M. Puig, N. Lagardera, F. (2009). Sociología del deporte. Madrid: Alianza.

Guichard, C. (2015). Manual de comunicación no sexista. Hacia un lenguaje incluyente. México: INMUJERES

INMUJERES y CONADE. (2015) Mujer y deporte. Una visión de género. Recuperado http://cedoc.inmujeres.gob.mx/documentos_download/ 100559.pdf.

Isorna, M., Felpeto, M. Alonso, D., Gómez, P., \& Rial, A. (2019). Mujer y piragua: estudio de las variables moduladoras del abandono deportivo de las mujeres piragüistas en modalidades olímpicas. RETOS. Nuevas tendencias en Educación Física, Deporte y Recreación,35(1), 320-325.

Lamas, M. (1996). La perspectiva de género. Recuperado de http:// www.dgespe.sep.gob.mx/public/genero/PDF/LECTURAS/ S_01_13_La\%20perspectiva\%20de\%20g\%C3\%A9nero.pdf

Merino, M. (2005). La importancia de las rutinas: Marco teórico para una investigación sobre la gestión municipal en México. México: CIDE.

Martín, M., Moscoso, D. y Pedrajas, N. (2013). Diferencias de género en las motivaciones para practicar actividades físico-deportivas en la vejez. $R e$ vista Internacional de Medicina y Ciencias de la Actividad Física y el Deporte vol. 13 (49) pp.121-129. Recuperado de Http://cdeporte.rediris.es/ revista/revista49/artdiferencias350.htm

Nuviala, A., Grao, A., Fernández, A., Alda, O., Burges, J.A. \& Jaume, A. (2009). Autopercepción de la salud, estilo de vida y actividad física organizada. Revista Internacional de Medicina y Ciencias de la Actividad Física y el Deporte vol. 9 (36) pp. 414-430. Recuperado de Http:// cdeporte.rediris.es/revista/revista36/artsalud121.htm

Pérez, K. (s/f). Más allá de la igualdad formal: Dignidad humana y combate a la desventaja. IIJ UNAM. Recuperado de https:// archivos.juridicas.unam.mx/www/bjv/libros/6/2834/27.pdf

Pérez, S., Castaño, R., Sánchez, A., Rodríguez, A., De Mena, J., Fuentes, J., \& Castaño, C. (2018). ¿Por qué juego al fútbol si soy una mujer?: Motivaciones para jugar al fútbol. RETOS. Nuevas tendencias en Educación Física, Deporte y Recreación,34 (2), 183-188.

Postigo, M. (2005). La conquista de la igualdad y las acciones afirmativas: de la igualdad formal a la igualdad sustancial. CLEPSYDRA, (4), 33-45.

Programa Nacional de Cultura Física y Deporte 2013- 2018.

Puig, N.\& Soler, S. (2004). Mujer y Deporte en España: estado de la cuestión y propuesta interpretativa. Apunts. Educación Física y Deportes (76), 7178.

Rodríguez, L. \& Miraflores, E. (2018). Propuesta de igualdad de género en Educación Física: adaptaciones de las normas en fútbol. RETOS. Nuevas tendencias en Educación Física, Deporte y Recreación,33(1), 293-297.

Tarducci G, Barengo N, Morea G, Gárgano S, Gandini A, Paganini A, Bardach A. (2016). Relación entre el nivel de escolaridad y el patrón de actividad física en Balcarce, Argentina. Hacia promoc. salud. 21(2), 89-98. DOI: 10.17151/hpsal.2016.21.2.7

Valdez, F. (2015). Fomento de la actividad física en México Una política pública inacabada. Gestión y Política Pública, (Vol. Temático), 27-54.

Vargas, E. \& Gambara, H. (2012). Evaluación del grado de sensibilidad frente al enfoque de derechos humanos y la perspectiva de género. Madrid: Catarata. 